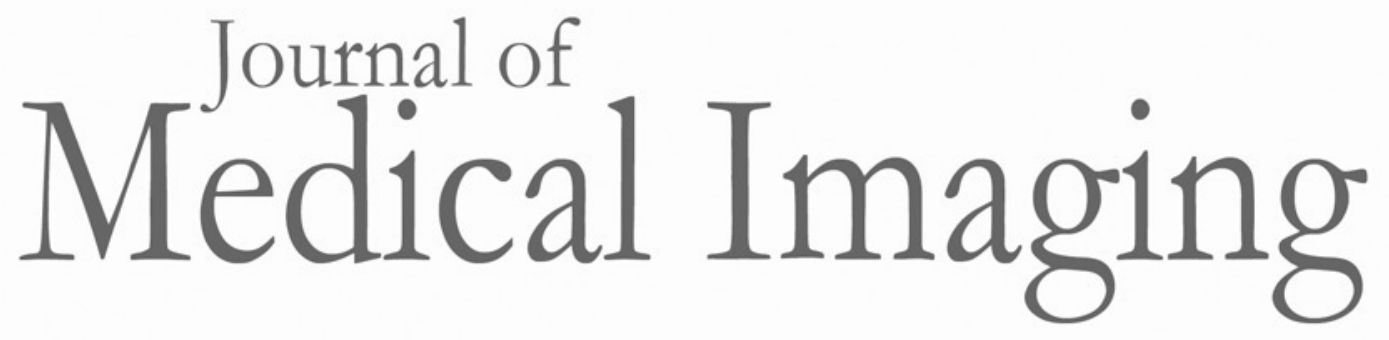

Medicallmaging.SPIEDigitalLibrary.org

\title{
Quantitative performance characterization of image quality and radiation dose for a CS 9300 dental cone beam computed tomography machine
}

Elham Abouei

Sierra Lee

Nancy L. Ford

\section{SPIE.}




\title{
Quantitative performance characterization of image quality and radiation dose for a CS 9300 dental cone beam computed tomography machine
}

\author{
Elham Abouei, ${ }^{a}$ Sierra Lee, ${ }^{b}$ and Nancy L. Ford ${ }^{a, b, *}$ \\ aniversity of British Columbia, Department of Physics and Astronomy, 6224 Agricultural Road, Vancouver, B.C. V6T 1Z1, Canada \\ bUniversity of British Columbia, Department of Oral Biological and Medical Sciences, 2199 Webrook Mall, Vancouver, B.C. V6T 1Z3, Canada
}

\begin{abstract}
This paper aims to characterize the radiation dose and image quality (IQ) performance of a dental cone beam computed tomography (CBCT) unit over a range of fields of view (FOV). IQ and dose were measured using a Carestream 9300 dental CBCT. Phantoms were positioned in the FOV to imitate clinical positioning. IQ was assessed by scanning a SEDENTEXCT IQ phantom, and images were analyzed in ImageJ. Dose index 1 was obtained using a thimble ionization chamber and SEDENTEXCT DI phantom. Mean gray values agreed within $93.5 \%$ to $99.7 \%$ across the images, with pixel-to-pixel fluctuations of $6 \%$ to $12.5 \%$, with poorer uniformity and increased noise for child protocols. CNR was fairly constant across FOVs, with higher CNR for larger patient settings. The measured limiting spatial resolution agreed well with $10 \%$ MTF and bar pattern measurements. Dose was reduced for smaller patient settings within a given FOV; however, smaller FOVs obtained with different acquisition settings did not necessarily result in reduced dose. The use of patient-specific acquisition settings decreased the radiation dose for smaller patients, with minimal impact on the IQ. The full set of IQ and dose measurements is reported to allow dental professionals to compare the different FOV settings for clinical use. (C) The Authors. Published by SPIE under a Creative Commons Attribution 3.0 Unported License. Distribution or reproduction of this work in whole or in part requires full attribution of the original publication, including its DOI. [DOI: 10.1117/1.JMI.2.4.044002]
\end{abstract}

Keywords: image quality; image evaluation; computer tomography; imaging.

Paper 15098RRR received May 6, 2015; accepted for publication Oct. 16, 2015; published online Nov. 18, 2015.

\section{Introduction}

Cone beam computed tomography (CBCT) has been used in dentistry since the early 2000 s in North America. Dental CBCT machines are primarily installed in private dental offices, but guidelines for the use and maintenance of these machines have not been widely adopted. ${ }^{1}$ Early investigations compared the image quality (IQ) of dental CBCT machines with multislice computed tomography (MSCT) to identify the utility of the dental devices. Subjective observer assessments of the IQ and visibility of structures have been reported, where the observers scored images of a dry skull obtained with both dental CBCT and MSCT scanners, showing that dental CBCT units provide adequate IQ for dentomaxillofacial radiology. ${ }^{2}$ Using a phantom provided by the dental CBCT vendor, Loubele et al. ${ }^{3}$ compared the contrast-to-noise ratio (CNR), mean gray values, and segmentation accuracy between CBCT and MSCT, with the conclusion that the dental unit proved better for bone segmentation and was less prone to artifacts resulting from metal appliances. The same researchers also investigated the dose efficiency of dental CBCT by correlating the segmentation efficacy with the measured dose. ${ }^{4}$ Suomalainen et al. ${ }^{5}$ measured the CNR and spatial resolution by placing test objects in a radiosurgery verification phantom and compared the IQ for two MSCT and four CBCT machines, determining that adequate images could be obtained but optimizing the acquisition parameters would be important. Dillenseger et al. ${ }^{6}$ compared three MSCT scanners with a dental system using a quality assurance phantom ${ }^{7}$ developed for

${ }^{\star}$ Address all correspondence to: Nancy L. Ford, E-mail: nlford@ dentistry.ubc.ca microcomputed tomography and concluded that the IQ achieved was comparable between the machines, although the uniformity across the FOV was better for the MSCT scanners.

To create a quality assurance program that could be adopted into routine clinical practice requires standardized measurement tools and a framework for their use. Steiding et al. ${ }^{8}$ have developed a framework for quality assurance testing, including an IQ phantom and automated analysis software. The proposed phantom and software have been tested on a dental CBCT unit, although they are proposed for widespread use in CBCT machines in radiology, in addition to the dental units. In Europe, the Sedentex consortium developed a pair of phantoms, one for measuring the IQ and one to measure the dose to the patient. ${ }^{9}$ The Sedentex IQ phantom has been used to assess the correlation between objective IQ metrics and clinical diagnostic value using a skull phantom. ${ }^{10}$ To address the requirement that the dental professionals perform quality assurance testing during their regular clinic hours, a user-friendly and timeefficient system was developed, with setup, imaging, and automated software analysis completed in approximately 10 min. ${ }^{11}$

Having the standardized tools in place is a step toward an effective quality assurance program. However, criteria for the measured IQ must be developed to ensure that machines are operating within the recommended framework. To date, there is no large-scale audit reported for assessing the IQ over the full range of operating parameters for any of the installed CBCT models. ${ }^{1}$ To create appropriate IQ criteria for a quality assurance program, baseline values for the important IQ metrics must be measured for a variety of equipment models and imaging settings. In this study, we use the Sedentex phantoms to 
measure the IQ and dose for the full range of fields of views (FOVs) and imaging parameters for different patient demographics (child, small, average, and large adults) to characterize the Carestream 9300 system.

\section{Materials and Methods}

\subsection{Phantoms and Image Acquisition}

In this study, the IQ and radiation dose of a dental CBCT machine (CS 9300, Carestream Dental LLC, Atlanta, Georgia) were evaluated using IQ and dose index (DI) phantoms developed by Leeds Test Objects Ltd. (SEDENTEXCT, Boroughbridge, UK) for dental CBCT machines. Both phantoms are head-sized cylinders $(160-\mathrm{mm}$ diameter by $162-\mathrm{mm}$ high) made of poly-methyl methacrylate (PMMA) with a density of $1.20 \pm 0.01 \mathrm{~g} / \mathrm{cm}^{3}$ to simulate the human tissue. ${ }^{9}$ Each phantom was placed on a tripod, leveled with a spirit level, and positioned using the lasers to align the phantom within the FOV, as shown in Fig. 1. IQ assessment was obtained using images of the IQ phantom scanned with the parameters listed in Table 1 and analyzed using ImageJ (National Institutes of Health, Bethesda, Maryland) software. To evaluate the radiation dose, the DI phantom was scanned with the same imaging parameters (Table 1). A calibrated thimble ionization chamber system, a $0.6-\mathrm{cm}^{3}$ Farmer ionization chamber $(10 \times 60.6 \mathrm{CT}$, Radcal Corp., Monrovia, California) in conjunction with a Radcal electrometer (AccuDose, Radcal Corp., Monrovia, California), was used following the SEDENTEX dose index (DI1) methodology. XR-QA2 ${ }^{\circledR}$ Gafchromic ${ }^{\text {TM }}$ (ISP Technologies Inc., Wayne, New Jersey) films were used to visualize axial dose distribution.

The dental CBCT machine is the Carestream 9300 (Carestream Dental LLC, Atlanta, Georgia), which uses a partial rotation about the patient during image acquisition.

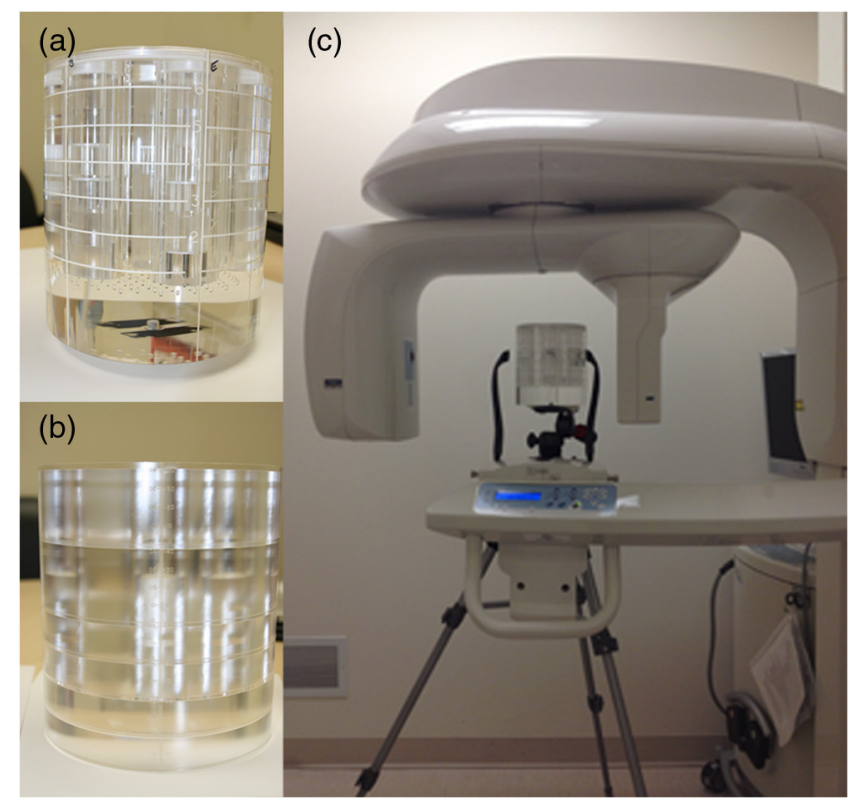

Fig. 1 The Sedentex phantoms (a) IQ for measuring image quality, (b) DI for measuring dose, and (c) the IQ phantom positioned on the tripod in the Carestream 9300 CBCT machine. The lines on the exterior of the IQ phantom indicate the different layers containing test objects. The DI phantom has holes drilled through for ion chamber placement, and PMMA plugs to fill unused holes.
This machine has a variety of cylindrical imaging FOV that cover the craniofacial complex $(17-\mathrm{cm}$ diameter $\times 11-\mathrm{cm}$ height, 17 -cm diameter $\times 6$-cm height), the maxillofacial area $(10-\mathrm{cm}$ diameter $\times 10$-cm height, 10 -cm diameter $\times 5$-cm height $)$, and for imaging individual teeth $(8$-cm diameter $\times 8$-cm height, 5 -cm diameter $\times 5$-cm height). The machine comes with preinstalled imaging protocols for use with different patient demographics, including children, small adults, average adults, and large adults (Table 1), with the option of a high-resolution or low-resolution mode for each. The high-resolution modes offer improved resolution in the images, albeit with increased scan times and x-ray dose to the patient. For this paper, we investigate all patient sizes with voxel sizes of 180 to $250 \mu \mathrm{m}$ to enable comparisons between the protocols, although voxel sizes of $90 \mu \mathrm{m}$

Table 1 Factory installed protocols on the CS 9300 СBCT machine. Each patient setting was preprogrammed by the manufacturer, varying the $\mathrm{kVp}, \mathrm{mA}$, and reconstructed voxel size for each patient size. These are the settings that are used clinically.

\begin{tabular}{|c|c|c|c|c|c|}
\hline $\begin{array}{l}\text { FOV } \mathrm{cm}^{2} \\
\text { (diameter } \times \text { height) }\end{array}$ & Patient type & $k V p$ & $\mathrm{~mA}$ & $\begin{array}{c}\text { Time } \\
(\mathrm{s})\end{array}$ & $\begin{array}{l}\text { Voxel } \\
(\mu \mathrm{m})\end{array}$ \\
\hline \multirow[t]{4}{*}{ FOV $17 \times 11$} & Child & 80 & 4 & 6.40 & 250 \\
\hline & Adult small & 85 & 4 & 6.40 & 250 \\
\hline & Adult average & 90 & 4 & 6.40 & 250 \\
\hline & Adult large & 90 & 5 & 6.40 & 250 \\
\hline \multirow[t]{4}{*}{ FOV $17 \times 6$} & Child & 80 & 4 & 6.20 & 200 \\
\hline & Adult small & 85 & 4 & 6.30 & 200 \\
\hline & Adult average & 90 & 4 & 6.30 & 200 \\
\hline & Adult large & 90 & 5 & 6.30 & 200 \\
\hline \multirow[t]{4}{*}{ FOV $8 \times 8$} & Child & 80 & 4 & 8.00 & 180 \\
\hline & Adult small & 85 & 4 & 8.00 & 180 \\
\hline & Adult average & 90 & 4 & 8.00 & 180 \\
\hline & Adult large & 90 & 5 & 8.00 & 180 \\
\hline \multirow[t]{4}{*}{ FOV $10 \times 10$} & Child & 80 & 4 & 6.20 & 180 \\
\hline & Adult small & 85 & 4 & 6.20 & 180 \\
\hline & Adult average & 90 & 4 & 6.20 & 180 \\
\hline & Adult large & 90 & 5 & 6.20 & 180 \\
\hline \multirow[t]{4}{*}{ FOV $10 \times 5$} & Child & 80 & 4 & 6.20 & 180 \\
\hline & Adult small & 85 & 4 & 6.20 & 180 \\
\hline & Adult average & 90 & 4 & 6.20 & 180 \\
\hline & Adult large & 90 & 5 & 6.20 & 180 \\
\hline \multirow[t]{4}{*}{ FOV $5 \times 5$} & Child & 75 & 4 & 12 & 200 \\
\hline & Adult small & 80 & 5 & 12 & 200 \\
\hline & Adult average & 84 & 5 & 12 & 200 \\
\hline & Adult large & 84 & 8 & 12 & 200 \\
\hline
\end{tabular}


are possible for the smallest FOV $(5 \times 5 \mathrm{~cm})$ and voxel sizes of $500 \mu \mathrm{m}$ are possible for the largest FOV $(17 \times 11 \mathrm{~cm})$.

\subsection{Image Quality Measurements}

The IQ phantom has seven vertical columns drilled through the PMMA, which are filled with different test objects to assess IQ. Each hole is $35 \mathrm{~mm}$ in diameter, with one in the center of the phantom and the others equally spaced around the periphery. The complete design of the phantom has been published by Pauwels et al. ${ }^{9}$ Each set of test objects is placed at a different height within the phantom to obtain all of the IQ data simultaneously. To assess the IQ, the center slice for each test object was located in the orthogonal view, and five adjacent slices were used for the measurements (two above, the central slice and two below). By using only the middle five slices of the test objects, the measurements were less sensitive to misalignment of the phantom, partial volume at the edges, or artifacts from adjacent test objects.

To measure the image noise, five circular ROIs were placed at the center (ROI 5) and periphery (ROIs 1, 2, 3, 4) of the FOV on the center slice of noise/uniformity layer oriented as shown in Fig. 2(a). ROIs were $40 \mathrm{~mm}$ in diameter for images obtained with the $17-\mathrm{cm}$ diameter FOVs, and the ROI diameter was $20 \%$ of the FOV diameter for all other FOV sizes. Noise was defined as average SD of gray values of five ROIs. The SD was normalized to the difference of MGV between PMMA and air regions [Eq. (1) $]^{12}$ to be comparable through different protocols, since the mean values for each protocol were different. Uniformity was defined as the difference between MGV of central and peripheral ROIs, as given by Eq. (2). ${ }^{12} \mathrm{MGV}$ and SD were measured on images of the lower part of the IQ phantom that had uniform PMMA across the entire phantom diameter. Uniformity was also assessed by vertical and horizontal line profiles through the center of the central slice. Care was taken to ensure that the lines drawn were orthogonal to each other and passed through the center of the phantom (along the diameter)

Noise $=\frac{\mathrm{SD}}{\left(\mathrm{MGV}_{\mathrm{PMMA}}-\mathrm{MGV}_{\mathrm{Air}}\right)}$,

Uniformity $=\left(1-\left|\frac{\mathrm{MGV}_{\text {Center }}-\mathrm{MGV}_{\text {Peripheral }}}{\mathrm{MGV}_{\text {PMMA }}-\mathrm{MGV}_{\text {Air }}}\right|\right) \times 100$.

Periodicity of the evenly spaced voids in the geometric distortion layer was defined as the geometric distortion parameter.
It is measured using line profiles drawn manually across two perpendicular lines on the center slice of geometric distortion layer shown in Fig. 2(b). The actual spacing between the voids in the phantom is $10.0 \mathrm{~mm}$, with each void measuring $2.0 \mathrm{~mm}$ in diameter and 3.0-mm deep. The center-to-center distance between adjacent holes was calculated from the line profile and compared to the nominal value. The average centerto-center distance is reported.

For the quantitative evaluation of metal artifacts, the artifact added value (AAV) using two metal artifact inserts was calculated using Eq. (3). ${ }^{13}$ The AAV for each protocol was reported as the average of AAVs for the four ROIs shown in Fig. 2(c). Regions of interest near the metal inserts were chosen to extend the length of the three inserts but exclude the metal, and the mean gray value was obtained. Measurements of the mean gray value in PMMA and air were obtained in regions of interest in other layers and subtracted to yield the AAV

$$
\mathrm{AAV}=\frac{\mathrm{MGV}_{\text {Artifact }}-\mathrm{MGV}_{\text {PMMA }}}{\mathrm{MGV}_{\text {PMMA }}-\mathrm{MGV}_{\text {Air }}}
$$

The CNR was defined as the ratio of the difference between the MGV of PMMA and a material (Al, PTFE, POM, LDPE, and Air) to the average of SD of both PMMA and the material [Eq. (4)]. ${ }^{12} \mathrm{MGV}_{\text {PMMA }}$ and $\mathrm{SD}_{\text {PMMA }}$ are measured from the noise/uniformity layer, and $\mathrm{MGV}_{\text {material }}$ and $\mathrm{SD}_{\text {material }}$ are measured for 20-mm diameter circular ROI at each corresponding disk using the pixel intensity insert. The materials included in the phantom represent a range of densities consistent with human tissues, ranging between $0.90 \mathrm{~g} / \mathrm{cm}^{3}$ for LDPE, $1.18 \mathrm{~g} / \mathrm{cm}^{3}$ for PMMA, $1.41 \mathrm{~g} / \mathrm{cm}^{3}$ for POM, $2.2 \mathrm{~g} / \mathrm{cm}^{3}$ for PTFE, and $2.70 \mathrm{~g} / \mathrm{cm}^{3}$ for $\mathrm{Al}$.

$\mathrm{CNR}=\frac{\left|\mathrm{MGV}_{\text {Material }}-\mathrm{MGV}_{\text {PMMA }}\right|}{1 / 2\left(\mathrm{SD}_{\text {Material }}+\mathrm{SD}_{\text {PMMA }}\right)}$

The resolution $(\mathrm{LP} / \mathrm{mm})$ inserts were used to determine the spatial resolution of the image visually (Fig. 3). The smallest LP/ $\mathrm{mm}$ was visually counted for both the $x-y$-plane and $z$-axis inserts. The spatial resolution was also calculated quantitatively using the point spread function (PSF) insert, which contains a $0.25-\mathrm{mm}$ diameter stainless steel wire suspended in air parallel to the $z$-axis, to calculate the modulation transfer function (MTF) using MATLAB (Mathworks, Natick, Massachusetts). Ten adjacent central axial slices were averaged to generate a low-noise PSF, ensuring that the location of the wire remained constant through all slices used. A rectangular ROI, $30 \times$ 30 pixels, was centrally placed at the PSF insert. Pixel values
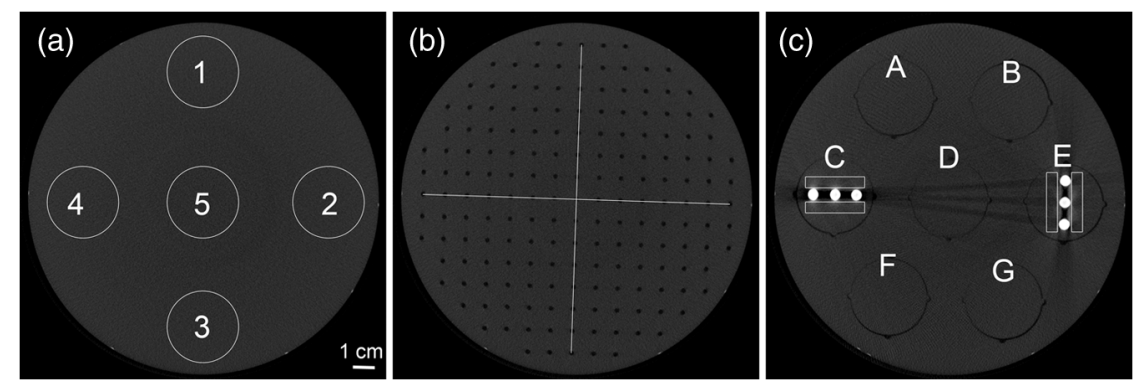

Fig. 2 (a) ROls for noise/uniformity, (b) two perpendicular lines to measure line profiles for geometric distortion, and (c) rectangular ROls around the artifact rods. 


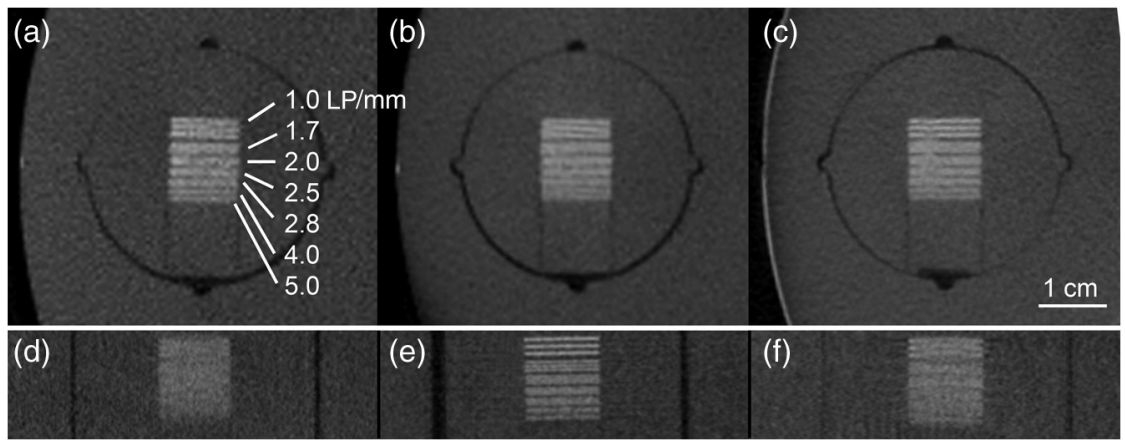

Fig. 3. The bar pattern insert imaged in the axial plane with voxel spacings (a) $250 \mu \mathrm{m}$, (b) $200 \mu \mathrm{m}$, and (c) $180 \mu \mathrm{m}$. The images for z-axis are shown in (d), (e), and (f) respectively. The bar pattern spacing ranges from 1.0 to $5.0 \mathrm{LP} / \mathrm{mm}$.

over this ROI were integrated in the longitudinal direction (integrating along either the $x$ - or $y$-axis) to yield a one-dimensional (1-D) PSF. The resulting distribution was plotted, and full-width at half-maximum (FWHM) was calculated. The MTF was calculated from 1-D PSF using the fast Fourier transform method, and divided by the spatial frequency spectrum described by the steel wire. ${ }^{14,15}$ The frequency at $10 \%$ of the MTF was used to calculate the limiting spatial resolution (Fig. 4), as this provides the limiting resolution for a human observer that can be compared to the subjective measurement of the bar pattern insert. The limiting resolution is calculated using Eq. (5), where $\mathrm{LP}_{10 \% \mathrm{MTF}}$ is the frequency at $10 \% \mathrm{MTF}$

resolution $=\frac{1}{2 * \mathrm{LP}_{10 \% \mathrm{MTF}}}$.

(a)

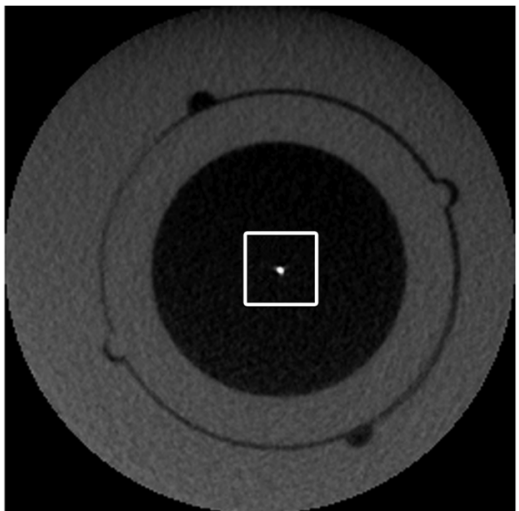

(b)

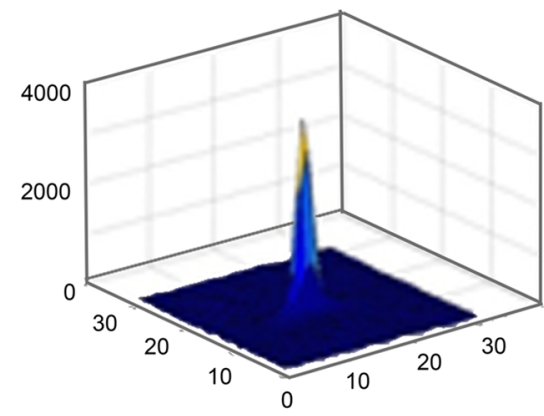

(c)

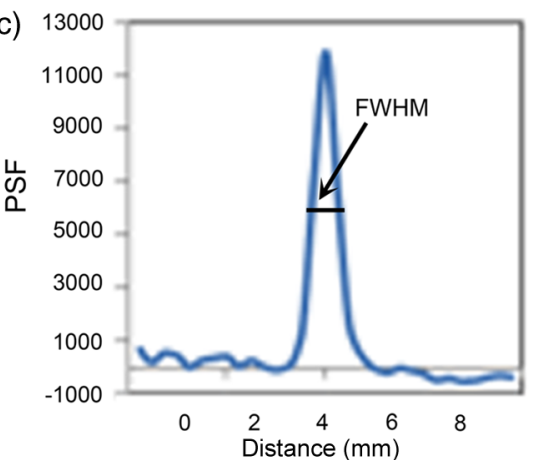

\subsection{Dose Measurement}

The SEDENTEXCT DI phantom, composed of a stack of six PMMA plates forming a cylinder of the size of an adult head, was scanned in the same configuration as a patient. Dose measurements were performed with the method adapted from the SEDENTEXCT project along a diameter of the phantom. ${ }^{16}$ The measuring diameter was determined from a dose distribution map generated using Gafchromic ${ }^{\mathrm{TM}}$ film placed on the center slice of the phantom. The film was scanned five times to accumulate sufficient dose to be in the sensitive range of the film, then it was scanned in a flatbed HP scanner (HP LJ 1536dnf MFP, Hewlett Packard Company, Palo Alto, California) and the dose distribution map obtained using MATLAB (Mathworks, Natick, Massachusetts). For asymmetric dose distributions, a line profile passing through the center of

Fig. 4 (a) The ROI on the central axial slice of the point spread function (PSF) insert. (b) Surface plot of the PSF insert over the ROI. (c) An integrated one-dimensional (1-D) profile of the PSF. (d) MTF obtained from the FFT of the 1-D PSF. 
(a)
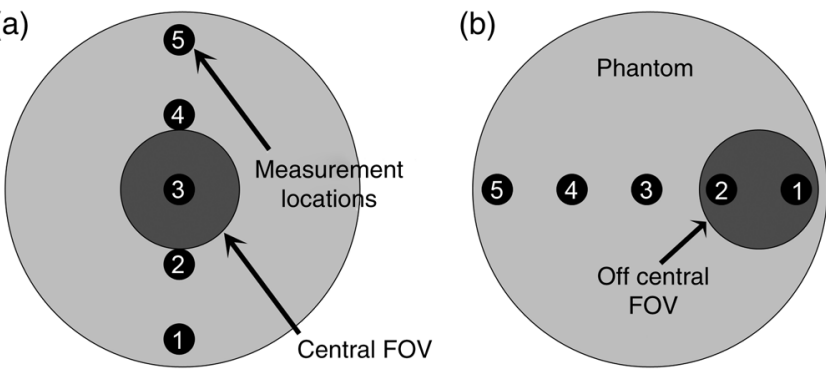

Fig. 5 The dose measurement points for DI1 using DI phantom. Number 1 is located at interior/right side of the phantom.

the phantom that exhibited the largest gradient in gray values was identified as the measurement diameter for the ion chamber placement. For symmetric dose distributions, any measurement along the diameter of the phantom should be sufficient. By determining the measuring diameter, dose measurements at five points along this line were performed using a thimble ionization chamber (Fig. 5), and then DI was defined as the average dose along this line [Eq. (6)]. Two scans at each point were taken to assess variation without overheating the $\mathrm{x}$-ray tube; these two dose values reported in milligray were averaged to give the radiation dose at each point

$\mathrm{DI} 1=\frac{\sum_{i=1}^{5} D_{i}}{5}$

\subsection{Variability of Image Quality Measurements}

For the largest $\left(17 \times 11 \mathrm{~cm}^{2}\right)$ and the smallest $\left(5 \times 5 \mathrm{~cm}^{2}\right)$ FOVs, intraobserver variability, interobserver variability, and interscan reproducibility of the images obtained from the CS 9300 machine were determined using the average adult settings to assess the consistency and reproducibility of the measurements. For interscan reproducibility, three phantom images were collected and analyzed by a single observer; the phantom was set up and taken down for each of the three phantom images. For intraobserver variability, a single observer analyzed the images on three different dates, 14 days apart. For interobserver variability, a second observer analyzed the same data. The standard deviation of measurements was calculated for the reproducibility and intraobserver variability consistency, and the difference between the measurements was recorded for the interobserver variability. For the remaining settings and FOVs, the phantom was imaged and analyzed once by a single observer.

\section{Results}

\subsection{Image Quality Parameters}

The IQ results for noise, uniformity, geometric distortion, and artifacts are summarized in Table 2. As shown in Fig. 6, the $17 \times$ $11 \mathrm{~cm}^{2}$ FOV had the greatest noise value and the $8 \times 8 \mathrm{~cm}^{2}$ FOV had the lowest noise value. The $5 \times 5 \mathrm{~cm}^{2}$ FOV had noise values less than $17 \times 11 \mathrm{~cm}^{2}$ FOV and larger than other FOVs, likely due to reduced $\mathrm{kVp}$ settings for the $5 \times 5 \mathrm{~cm}^{2}$ FOV. For all FOV options, the child settings had the highest noise indicating that fewer photons were reaching the detector, likely due to a reduction in the dose to the patient.
Table 2 Noise, uniformity, geometric distortion, and AAV obtained for different protocols on CS $9300 \mathrm{CBCT}$.

\begin{tabular}{|c|c|c|c|c|c|}
\hline $\begin{array}{l}\text { FOV } \mathrm{cm}^{2} \\
\text { (diameter } \times \\
\text { height) }\end{array}$ & Patient type & $\begin{array}{c}\text { Noise } \\
(\%)\end{array}$ & $\begin{array}{c}\text { Uniformity } \\
(\%)\end{array}$ & $\begin{array}{c}\text { Geometric } \\
\text { distortion } \\
(\mathrm{mm})\end{array}$ & AAV \\
\hline \multirow[t]{4}{*}{$17 \times 11$} & Child & 19.5 & 93.5 & 9.98 & 0.4 \\
\hline & Adult small & 17.9 & 93.9 & 9.97 & 0.4 \\
\hline & Adult average & 16.4 & 94.8 & 9.99 & 0.3 \\
\hline & Adult large & 13.7 & 96.9 & 10.01 & 0.3 \\
\hline \multirow[t]{4}{*}{$17 \times 6$} & Child & 9.9 & 98.5 & 9.97 & 0.3 \\
\hline & Adult small & 9.1 & 99.1 & 9.99 & 0.3 \\
\hline & Adult average & 8.2 & 99.7 & 9.98 & 0.2 \\
\hline & Adult large & 7.5 & 98.8 & 9.98 & 0.3 \\
\hline \multirow[t]{4}{*}{$8 \times 8$} & Child & 7.6 & 94.4 & 10.00 & 0.3 \\
\hline & Adult small & 7.3 & 95.6 & 10.00 & 0.3 \\
\hline & Adult average & 6.1 & 98.3 & 10.00 & 0.3 \\
\hline & Adult large & 6.4 & 98.4 & 10.05 & 0.3 \\
\hline \multirow[t]{4}{*}{$10 \times 10$} & Child & 9.5 & 93.6 & 10.01 & 0.3 \\
\hline & Adult small & 8.8 & 94.2 & 10.02 & 0.3 \\
\hline & Adult average & 8.2 & 94.3 & 10.04 & 0.3 \\
\hline & Adult large & 7.1 & 96.0 & 9.95 & 0.3 \\
\hline \multirow[t]{4}{*}{$10 \times 5$} & Child & 8.6 & 95.8 & 10.02 & 0.4 \\
\hline & Adult small & 8.6 & 95.9 & 10.02 & 0.4 \\
\hline & Adult average & 7.9 & 96.1 & 10.02 & 0.4 \\
\hline & Adult large & 7.1 & 98.5 & 10.01 & 0.3 \\
\hline \multirow[t]{4}{*}{$5 \times 5$} & Child & 12.5 & 97.5 & 10 & 0.4 \\
\hline & Adult small & 10.8 & 96.6 & 10 & 0.4 \\
\hline & Adult average & 9.4 & 96.6 & 10 & 0.4 \\
\hline & Adult large & 8.5 & 95.3 & 10 & 0.4 \\
\hline
\end{tabular}

The uniformity of the images calculated with Eq. (2) was better than $93.5 \%$ for all protocols (Table 2), indicating the difference between the central and peripheral ROIs was less than $7 \%$. Figure 7 shows that the line profiles were symmetric with reduced gray-scale values in the middle of the individual graphs for $17 \times 11 \mathrm{~cm}^{2}$ and $17 \times 6 \mathrm{~cm}^{2}$ FOVs. For the other FOVs, the uniformity graphs were asymmetric because the FOV includes less than the full phantom width, but showed reduced gray-scale values at the center of the phantom. A cupping artifact occurs at the center of an object due to beam hardening (x-rays with higher energies are more likely to pass through).

The average periodicity in the voids was between 9.95 and $10.05 \mathrm{~mm}$ for each setting (Table 2), suggesting $\pm 0.05 \mathrm{~mm}$ 


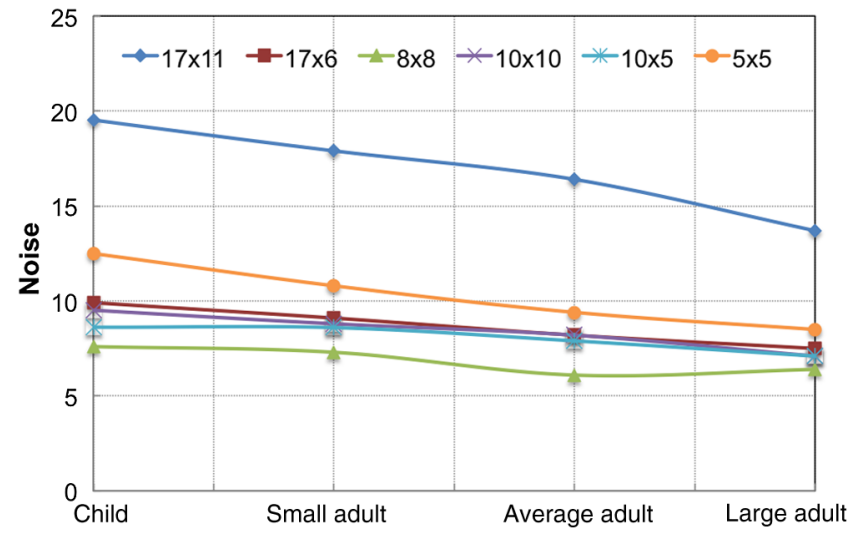

Fig. 6 Noise values for all FOVs and patient settings on CS 9300 machine.

error. The measurement error in each case is a fraction of the voxel spacing and agrees well with the actual spacing of $10.0 \mathrm{~mm}$.

The AAV was calculated from four rectangular ROIs per image and averaged (Table 2). The AAVs were between 0.3 and 0.4 for all protocols, except one protocol that had the value of 0.2. No clear difference was seen between large/small FOVs or high-/low-dose protocols.

To investigate the contrast, the MGV for each material in the pixel intensity insert is presented in Table 3. Aluminum (Al) had the greatest MGV value and air with the lowest value, as expected. The MGV values decreased for PTFE, POM, and LDPE, respectively. The standard deviations were greatest for the $\mathrm{Al}$ inserts for all protocols and were generally similar for the PTFE, POM, LDPE, and air materials. The CNRs were fairly consistent for each material for all the scanning protocols with the same FOV except for the small FOV, $5 \times 5 \mathrm{~cm}^{2}$, which had the greatest range of CNR values over different $\mathrm{mA}$ and $\mathrm{kVp}$ settings. These differences may occur because the images for each insert were acquired separately, and the range of grayscale values in the image may depend on the materials present in the FOV. Figure 8 shows the CNR value results for different protocols within each FOV. Al and air had the greatest and most similar CNR values, while there was a small difference between the CNR values of LDPE and POM values. The CNR was reduced for the smaller patient sizes (child, small adult), which was likely related to the increased noise for these acquisition settings.

The spatial resolution results are presented in Table 4. The FWHM were almost the same within each FOV but did not correlate with the reconstructed voxel size. Although $8 \times 8$, $10 \times 10$, and $10 \times 5 \mathrm{~cm}^{2}$ FOVs have the smallest reported voxel size $(180 \mu \mathrm{m})$, the FWHM was more than double the actual width of the wire $(250 \mu \mathrm{m})$. The results of the visual analysis of the line pair inserts in the $x-y$-plane and along the $z$-axis for each protocol were between 1 and $2 \mathrm{LP} / \mathrm{mm}$. Although the CBCT has isotropic voxels, the $\mathrm{LP} / \mathrm{mm}$ was not exactly the same in the $x-y$-plane and $z$-direction for most protocols, with better resolution observed in the axial (xy) plane. The limiting spatial resolution was calculated for each protocol from the $\mathrm{LP} / \mathrm{mm}$ measured at the $10 \%$ of the MTF graph and agreed well with the bar pattern measurements. As an example, Fig. 9 shows MTF curves for the child setting for six FOVs.

\subsection{Reproducibility of Image Quality Parameters}

The reproducibility of IQ parameters assessed through interscan, intraobserver, and interobserver variability represents the reproducibility of the phantom positioning, the measurement method, and the observer's analysis, respectively. Each IQ metric was measured as described earlier, and the standard deviation was taken of the repeated measurements to assess the variability. SD of the measurements obtained for interscan as well as intraobserver variability is shown in Table 5. The SD for CNR is the average $\mathrm{SD}$ of the CNRs of five materials.

Percentage difference between measurements obtained by two observers was reported as the interobserver variability in Table 6. The percentage difference for CNR is the percentage difference of the CNRs of all five materials. For interobserver reproducibility, the variation was less for noise, CNR, and $\mathrm{LP} / \mathrm{mm}$ for the $5 \times 5$ compared to $17 \times 11 \mathrm{~cm}^{2} \mathrm{FOV}$; however, it was larger for uniformity, geometric distortion, and AAV for $5 \times 5$ compared to $17 \times 11 \mathrm{~cm}^{2}$ FOV. The highest reproducibility was seen for uniformity and noise; on the other hand, metal artifact had the most deviation due to the large difference
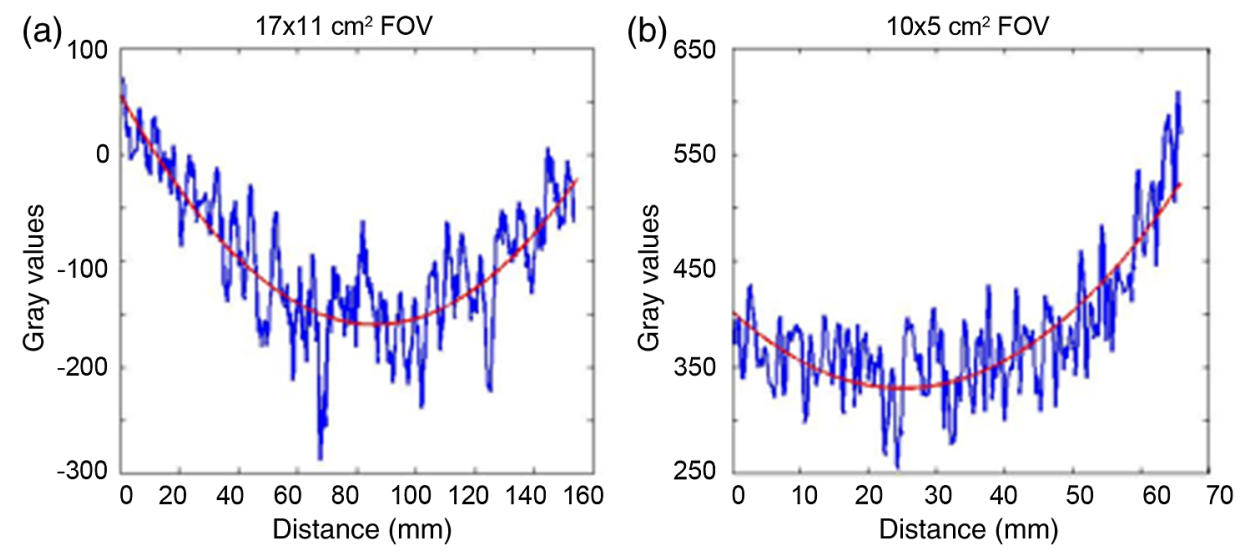

Fig. 7 The uniformity graphs including the noise in the image, which is not representing the actual noise in the image since it was smoothed for illustration purposes. (a) Uniformity profile at center of image representing symmetrical uniformity cupping at middle as an example for $17 \times 11$ (shown) and $17 \times 6 \mathrm{~cm}^{2}$ FOVs. (b) Uniformity profile at center of image is showing asymmetrical cupping uniformity at middle of image for $5 \times 5,8 \times 8,10 \times 10$, and $10 \times 5 \mathrm{~cm}^{2}$ (shown) FOVs. 
Table 3 Mean gray values and standard deviations for the different materials in the pixel intensity insert are reported for different protocols on CS 9300 CBCT.

\begin{tabular}{|c|c|c|c|c|c|c|}
\hline FOV $\mathrm{cm}^{2}$ (diameter $\times$ height) & Patient type & $\mathrm{Al}$ & PTFE & POM & LDPE & Air \\
\hline \multirow[t]{4}{*}{$17 \times 11$} & Child & $885 \pm 198$ & $382 \pm 141$ & $9 \pm 131$ & $-299 \pm 142$ & $-637 \pm 124$ \\
\hline & Adult small & $853 \pm 190$ & $403 \pm 130$ & $25 \pm 117$ & $-303 \pm 132$ & $-637 \pm 114$ \\
\hline & Adult average & $811 \pm 173$ & $382 \pm 118$ & $12 \pm 108$ & $-307 \pm 124$ & $-642 \pm 107$ \\
\hline & Adult large & $774 \pm 165$ & $305 \pm 105$ & $-54 \pm 99$ & $-351 \pm 117$ & $-698 \pm 102$ \\
\hline \multirow[t]{4}{*}{$17 \times 6$} & Child & $1549 \pm 211$ & $662 \pm 100$ & $160 \pm 81$ & $-195 \pm 70$ & $-944 \pm 57$ \\
\hline & Adult small & $1496 \pm 196$ & $637 \pm 91$ & $149 \pm 71$ & $-200 \pm 63$ & $-946 \pm 55$ \\
\hline & Adult average & $1437 \pm 182$ & $600 \pm 86$ & $120 \pm 65$ & $-223 \pm 57$ & $-957 \pm 49$ \\
\hline & Adult large & $1382 \pm 174$ & $547 \pm 74$ & $70 \pm 57$ & $-278 \pm 50$ & $-982 \pm 33$ \\
\hline \multirow[t]{4}{*}{$8 \times 8$} & Child & $2370 \pm 230$ & $1258 \pm 100$ & $649 \pm 84$ & $242 \pm 78$ & $-634 \pm 82$ \\
\hline & Adult small & $2160 \pm 196$ & $1132 \pm 89$ & $553 \pm 73$ & $143 \pm 68$ & $-688 \pm 76$ \\
\hline & Adult average & $2007 \pm 197$ & $997 \pm 85$ & $414 \pm 64$ & $22 \pm 60$ & $-828 \pm 65$ \\
\hline & Adult large & $1992 \pm 157$ & $980 \pm 72$ & $409 \pm 61$ & $18 \pm 60$ & $-815 \pm 74$ \\
\hline \multirow[t]{4}{*}{$10 \times 10$} & Child & $2031 \pm 241$ & $1088 \pm 118$ & $545 \pm 93$ & $176 \pm 82$ & $-626 \pm 84$ \\
\hline & Adult small & $1956 \pm 217$ & $1034 \pm 108$ & $495 \pm 90$ & $120 \pm 77$ & $-680 \pm 83$ \\
\hline & Adult average & $1769 \pm 197$ & $922 \pm 101$ & $392 \pm 81$ & $-10 \pm 82$ & $-701 \pm 85$ \\
\hline & Adult large & $1701 \pm 195$ & $834 \pm 91$ & $323 \pm 69$ & $-27 \pm 61$ & $-801 \pm 66$ \\
\hline \multirow[t]{4}{*}{$10 \times 5$} & Child & $2287 \pm 192$ & $1142 \pm 103$ & $538 \pm 92$ & $113 \pm 80$ & $-771 \pm 78$ \\
\hline & Adult small & $2398 \pm 208$ & $1219 \pm 117$ & $600 \pm 102$ & $177 \pm 88$ & $-723 \pm 85$ \\
\hline & Adult average & $2285 \pm 189$ & $1141 \pm 103$ & $537 \pm 92$ & $125 \pm 82$ & $-770 \pm 78$ \\
\hline & Adult large & $2210 \pm 191$ & $1062 \pm 94$ & $466 \pm 81$ & $43 \pm 72$ & $-852 \pm 74$ \\
\hline \multirow[t]{4}{*}{$5 \times 5$} & Child & $666 \pm 133$ & $55 \pm 77$ & $-254 \pm 67$ & $-447 \pm 61$ & $-862 \pm 56$ \\
\hline & Adult small & $586 \pm 106$ & $-5 \pm 58$ & $-304 \pm 52$ & $-494 \pm 49$ & $-902 \pm 45$ \\
\hline & Adult average & $512 \pm 88$ & $-47 \pm 50$ & $-338 \pm 46$ & $-528 \pm 44$ & $-931 \pm 41$ \\
\hline & Adult large & $512 \pm 83$ & $-73 \pm 42$ & $-365 \pm 37$ & $-558 \pm 34$ & $-949 \pm 33$ \\
\hline
\end{tabular}

between gray values in the ROI, and sensitivity of the rectangular ROI's positioning near the titanium rods [Fig. 2(c)].

\subsection{Dose Measurement}

Dose distributions for all six protocols are shown in Fig. 10, where the color map represents the percentage of the maximum dose, ranging from $0 \%$ shown as white to $100 \%$ shown as black. Different acquisition settings with the same FOV had very similar dose distributions on the axial slice. The measuring diameter for each FOV is shown by the line in Fig. 10, showing the location of the points shown in Fig. 3 used for the thimble ion chamber measurements. Point dose measurements along the measuring diameter are provided in Fig. 11 for the average adult protocol.
The DI1 ranged from 2.75 to $4.13 \mathrm{mGy}$ for large adults, 1.82 to $3.23 \mathrm{mGy}$ for average adults, 1.63 to $2.69 \mathrm{mGy}$ for small adults, and 1.01 to $2.21 \mathrm{mGy}$ for the child setting. Variability in the measurements was within $0.02 \mathrm{mGy}$ for all ion chamber positions in all FOVs. DI1 for each protocol is shown in Fig. 12 as column bars. The error bars represent the variability across the phantom for each FOV, with the largest error bars corresponding to the FOVs with a linear gradient in the dose distributions (Fig. 10). In general, DI1 increased for larger FOVs, higher $\mathrm{mA}$ and $\mathrm{kVp}$, smaller voxel sizes, and longer scan times, as expected. However, Fig. 12 also shows the $5 \times 5 \mathrm{~cm}^{2}$ FOV, large adult $(8 \mathrm{~mA}, 84 \mathrm{kV}, 12 \mathrm{~s}$, and $200 \mu \mathrm{m})$ in the far right column with a higher dose compared to the $17 \times 11 \mathrm{~cm}^{2}$ FOV, child (4 mA, $80 \mathrm{kV}, 6.40 \mathrm{~s}, 250 \mu \mathrm{m})$ shown in the leftmost column, making dose trends more difficult to predict across different FOVs. 
(a)

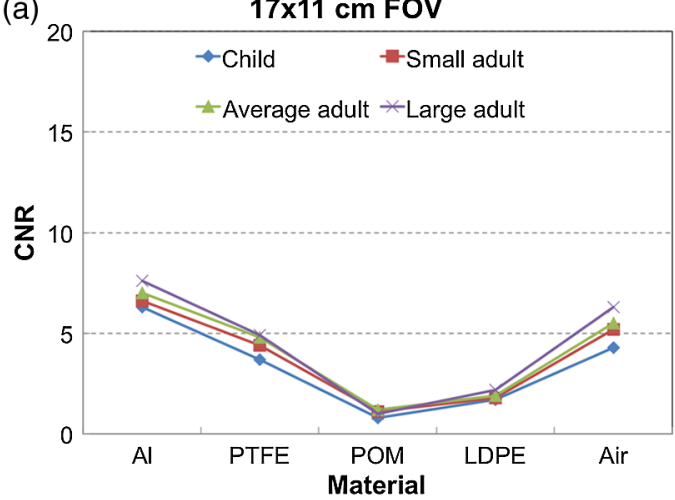

(c)

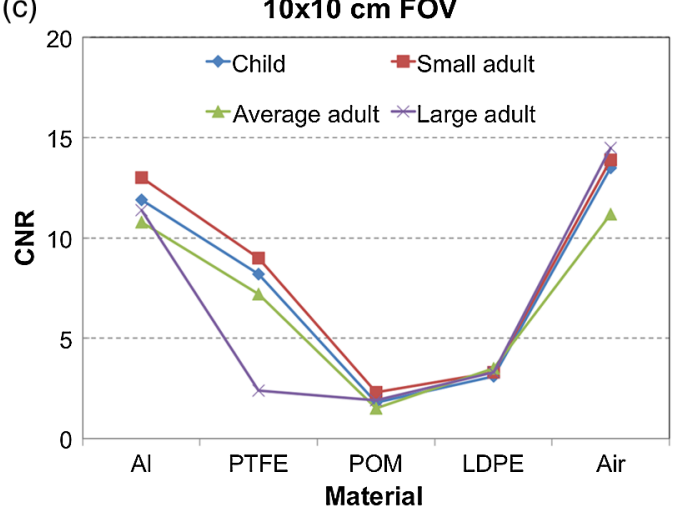

(e)

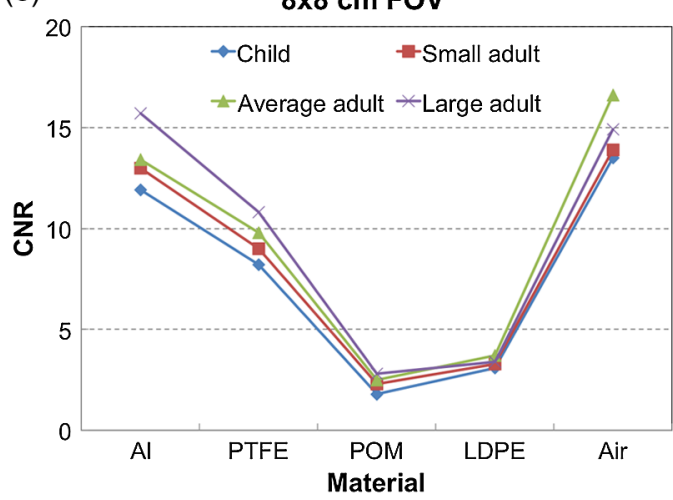

(b)

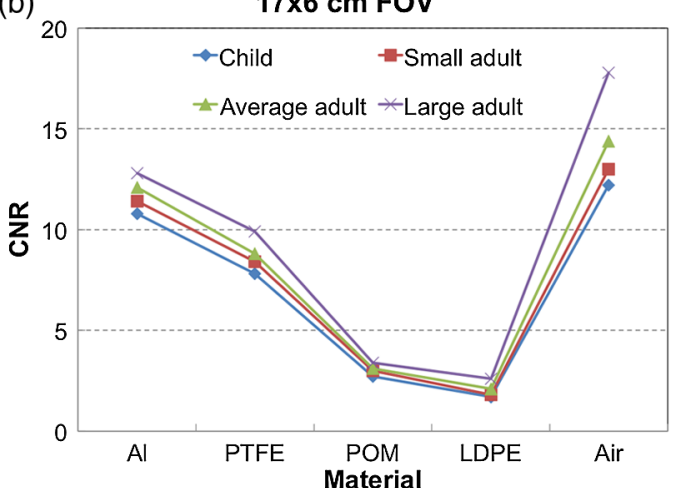

(d)

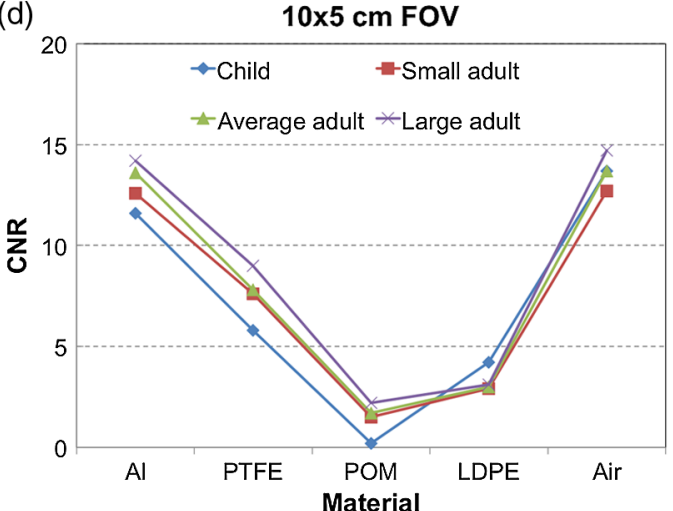

(f)

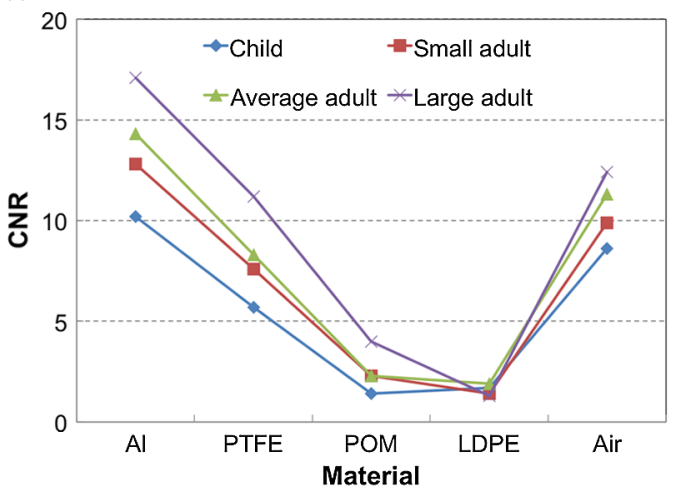

Fig. 8 The CNR values are plotted for the different materials in the phantom for each FOV: (a) $17 \times 11 \mathrm{~cm}^{2}$, (b) $17 \times 6 \mathrm{~cm}^{2}$, (c) $10 \times 10 \mathrm{~cm}^{2}$, (d) $10 \times 5 \mathrm{~cm}^{2}$, (e) $8 \times 8 \mathrm{~cm}^{2}$, and (f) $5 \times 5 \mathrm{~cm}^{2}$. Each graph shows four curves, which correspond to child (blue), small adult (red), average adult (green), and large adult (purple).

\section{Discussion}

Quality assurance in dental radiology is to ensure that consistently adequate diagnostic information is acquired, while the radiation dose is kept as low as reasonably achievable (ALARA). The SEDENTEXCT project recommended that the QA program be mainly composed of six subjects including performance of the $\mathrm{x}$-ray tube and generator, quantitative assessment of IQ, display screen performance, patient dose assessment, clinical IQ assessment, and clinical audit. ${ }^{16}$ Image intensity values, uniformity, and artifacts are recommended for monthly testing, and the others are recommended for yearly testing. ${ }^{15}$ It is important to select the CBCT exposure parameters considering both the radiation dose, adhering to the ALARA principle, and IQ evaluation.
In this study, a CS 9300 machine for application in dental imaging (maxillofacial region) was assessed in terms of technical performance (dose and IQ including noise, uniformity, geometric distortion, metal artifact, contrast resolution, and spatial resolution) for all FOVs and patient demographics. Reproducibility of the measurements of IQ parameters was assessed, and all measurements of the IQ parameters were proven to be reproducible within a few percent for all metrics (up to $30 \%$ for artifacts) in consecutive scans (interscan), as well as for repeated measurements of identical scans (interobserver or intraobserver). It can be expected that consecutive scans differ slightly in terms of voxel values due to slight variability in tube output, ${ }^{9}$ detector operation, and phantom positioning. Our measurements showed that the interscan reproducibility exhibited 
Table 4 Spatial resolution obtained from the full-width at half-maximum (FWHM) of the point spread function (PSF), the limiting resolution calculated using Eq. (5) from 10\% MTF and the bar pattern insert are reported for different protocols on CS 9300 CBCT.

\begin{tabular}{|c|c|c|c|c|c|c|}
\hline FOV $\mathrm{cm}^{2}$ (diameter $\times$ height) & Patient type & $\mathrm{FWHM}(\mathrm{mm})$ & $\begin{array}{l}\text { Spatial resolution } \\
(\mathrm{mm})\end{array}$ & $\begin{array}{c}10 \% \mathrm{MTF} \\
\text { (axial) }(\mathrm{LP} / \mathrm{mm})\end{array}$ & $\begin{array}{c}\text { Bar pattern } \\
\text { (axial) }(\mathrm{LP} / \mathrm{mm})\end{array}$ & $\begin{array}{c}\text { Bar pattern } \\
(z \text {-axis) }(\mathrm{LP} / \mathrm{mm})\end{array}$ \\
\hline \multirow[t]{4}{*}{$17 \times 11$} & Child & 0.59 & 0.22 & 2.2 & 1 & 0 \\
\hline & Adult small & 0.59 & 0.23 & 2.2 & 1 & 0 \\
\hline & Adult average & 0.56 & 0.21 & 2.4 & 1 & 0 \\
\hline & Adult large & 0.59 & 0.22 & 2.3 & 1 & 0 \\
\hline \multirow[t]{4}{*}{$17 \times 6$} & Child & 0.58 & 0.22 & 2.2 & 1 & 1.7 \\
\hline & Adult small & 0.58 & 0.22 & 2.3 & 1 & 1.7 \\
\hline & Adult average & 0.59 & 0.23 & 2.2 & 1 & 1.7 \\
\hline & Adult large & 0.57 & 0.22 & 2.3 & 1 & 1.7 \\
\hline \multirow[t]{4}{*}{$8 \times 8$} & Child & 0.66 & 0.27 & 1.8 & 1 & 1.7 \\
\hline & Adult small & 0.66 & 0.27 & 1.9 & 1 & 1.7 \\
\hline & Adult average & 0.58 & 0.25 & 2.0 & 1 & 1.7 \\
\hline & Adult large & 0.61 & 0.26 & 1.9 & 1.7 & 1.7 \\
\hline \multirow[t]{4}{*}{$10 \times 10$} & Child & 0.66 & 0.26 & 1.9 & 1.7 & 1 \\
\hline & Adult small & 0.67 & 0.26 & 1.9 & 1.7 & 1 \\
\hline & Adult average & 0.64 & 0.26 & 1.9 & 1.7 & 1 \\
\hline & Adult large & 0.67 & 0.26 & 1.9 & 1 & 1 \\
\hline \multirow[t]{4}{*}{$10 \times 5$} & Child & 0.65 & 0.26 & 1.9 & 1.7 & 1.7 \\
\hline & Adult small & 0.64 & 0.25 & 2.0 & 1.7 & 1.7 \\
\hline & Adult average & 0.64 & 0.25 & 2.0 & 1.7 & 1.7 \\
\hline & Adult large & 0.64 & 0.25 & 2.0 & 1.7 & 1.7 \\
\hline \multirow[t]{4}{*}{$5 \times 5$} & Child & 0.5 & 0.19 & 2.7 & 2 & 2 \\
\hline & Adult small & 0.52 & 0.19 & 2.6 & 2 & 2 \\
\hline & Adult average & 0.51 & 0.17 & 3.0 & 2 & 2.5 \\
\hline & Adult large & 0.5 & 0.19 & 2.6 & 2 & 2 \\
\hline
\end{tabular}

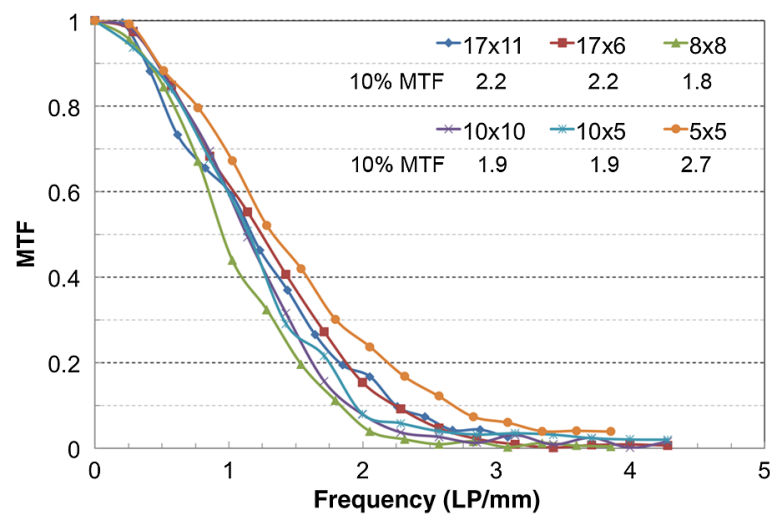

Fig. 9 The MTF graphs for the child setting for all six FOVs. more variability than interobserver/intraobserver reproducibility. For interscan reproducibility, the SDs for all IQ parameter values in the $5 \times 5 \mathrm{~cm}^{2}$ FOV were larger than the $17 \times 11 \mathrm{~cm}^{2}$ FOV, because more image acquisitions and more adjustments were required to image the entire phantom for the $5 \times 5 \mathrm{~cm}^{2}$ FOV. For intraobserver variability, the $5 \times 5 \mathrm{~cm}^{2}$ FOV had less variation than the $17 \times 11 \mathrm{~cm}^{2}$ FOV except for AAV parameter since the $5 \times 5 \mathrm{~cm}^{2}$ FOV was acquired with longer exposure time, which reduced the variability in the measurements performed by a single observer.

This study showed that image noise and uniformity values were not only affected by the exposure, but that the noise values for $17 \times 6 \mathrm{FOV}, 10 \times 10 \mathrm{FOV}$, and $10 \times 5 \mathrm{~cm}^{2} \mathrm{FOV}$ were comparable because they were all acquired with the same exposure times, $\mathrm{kVp}, \mathrm{mA}$, and voxel sizes. The location of the FOV and 
Table 5 Reproducibility and intraobserver variability of measurements. For interscan variability, three images were obtained and compared for each FOV. For intraobserver variability, the same image was assessed three times by a single observer.

\begin{tabular}{lccccc} 
& \multicolumn{2}{c}{$\begin{array}{c}\text { SD for interscan } \\
\text { variability }\end{array}$} & & \multicolumn{2}{c}{$\begin{array}{c}\text { SD for intraobserver } \\
\text { variability }\end{array}$} \\
\cline { 2 - 3 } \cline { 5 - 6 } $\begin{array}{l}\text { Image quality } \\
\text { parameter }\end{array}$ & $\begin{array}{c}5 \times 5 \mathrm{~cm}^{2} \\
\text { FOV }\end{array}$ & $\begin{array}{c}17 \times 11 \mathrm{~cm}^{2} \\
\text { FOV }\end{array}$ & & $\begin{array}{c}5 \times 5 \mathrm{~cm}^{2} \\
\text { FOV }\end{array}$ & $\begin{array}{c}17 \times 11 \mathrm{~cm}^{2} \\
\text { FOV }\end{array}$ \\
\hline Uniformity & 0.9 & 0.4 & & 0.02 & 0.1 \\
Noise & 0.7 & 0.7 & & 0.04 & 0.3 \\
$\begin{array}{l}\text { Geometric } \\
\text { distortion }\end{array}$ & 0.06 & 0.03 & & 0.06 & 0.08 \\
$\begin{array}{l}\text { Artifact } \\
\text { added value }\end{array}$ & 0.06 & 0.04 & & 0.1 & 0.05 \\
CNR & 0.4 & 0.2 & & 0.03 & 0.2 \\
LP/mm & 0.06 & 0.03 & & 0.02 & 0.04 \\
\hline
\end{tabular}

the amount of mass outside the volume were additional parameters leading to differences in performance; the "exo-mass" effect has been defined as the gradient of voxel values appearing for asymmetrical phantom positioning and causes a shift in density response throughout the entire volume, as well as a decrease in uniformity. ${ }^{9,17}$ Beam hardening and correction of projection data may also affect the image noise and uniformity. ${ }^{15}$

All protocols showed geometric distortion of less than $0.05 \mathrm{~mm}$; the geometric distortion is generally required to be less than $1 \mathrm{~mm}$ for a measurement to be considered accurate. ${ }^{18}$ The measurement error could be due to geometric distortion or the partial volume effect. ${ }^{19}$ There may also be minor misalignment of the rows of voids and the positioning aids on the exterior of the phantom, causing the voids to cross axial planes. Geometric distortions can be caused by detector misalignment with the imaging plane during the gantry rotation or nonuniformities in the detector material. Geometric distortions are rare in modern equipment and are generally eliminated by calibration adjustments or corrected in software. Artifacts arising from photon starvation in the vicinity of the titanium rods appeared similar comparing different protocols

Table 6 Interobserver variability.

\begin{tabular}{lcc}
\hline & \multicolumn{2}{c}{$\begin{array}{c}\% \text { Difference for } \\
\text { interobserver variability }\end{array}$} \\
\cline { 2 - 3 } Image quality parameter & $5 \times 5 \mathrm{~cm}^{2} \mathrm{FOV}$ & $17 \times 11 \mathrm{~cm}^{2}$ FOV \\
\hline Uniformity & 0.2 & 0.1 \\
Noise & 0.4 & 3 \\
Geometric distortion & 0.2 & 0.07 \\
Artifact added value & 30.5 & 10.8 \\
CNR & 1 & 2 \\
LP/mm & 0.4 & 6 \\
\hline
\end{tabular}

and was quantified by calculating the AAV, similar to the methods established by Pauwels et al. ${ }^{13}$ The AAV provides a relevant description of the net effect of streaks on IQ at the vicinity of the titanium rods, but provides no information regarding extinction, beam hardening, or other artifacts. Artifacts around metallic restoration materials are problematic, as CBCT is used for follow up in these patients, and the important regions to visualize (soft tissues, such as oral mucosa, and the underlying bone) are often adjacent to the metal. Artifacts in this region may interfere with assessing the integration of the metallic post into the surrounding bone, which is an important predictor of implant survival. The AAV values measured in this study seem to agree with the range provided by Pauwels et al., ${ }^{9}$ although the Carestream 9300 was not investigated in Pauwel's study.

Soft-tissue contrast resolution was somewhat limited, especially for the large FOV $\left(17 \times 11 \mathrm{~cm}^{2}\right)$. CNR measurements showed the general poor contrast for materials of intermediate density; the noise is often similar or larger than the measured difference in mean voxel value between a low-density material and PMMA. Dental CBCT is optimized for imaging highcontrast material, such as the bone and tooth structure in the maxillofacial region, and is typically used for planning oral surgeries and in the field of orthodontics. ${ }^{20}$ During planning, the dentist or surgeon will need to see important soft tissues in addition to bone and tooth, such as facial nerves, ligaments, infected areas, and pathologies such as cysts and tumors. Therefore, the soft tissue contrast is important to investigate for treatment planning and assessment as well as for identifying any suspicious lesions that may be present. Assessing the CNR and contrast resolution for a variety of tissue-like materials may predict at what size different types of tissues will be visible in the images.

The bar pattern test exhibited limiting resolutions of $1-2 \mathrm{LP} / \mathrm{mm}$ for all protocols, exhibiting acceptable resolution as suggested by Brullman and Schulze. ${ }^{21}$ The measured limiting spatial resolution did not correlate with reconstructed voxel size, which is consistent with the measurements of Pauwels et al. ${ }^{22}$ and Bamba et al. ${ }^{15}$ The images are resampled for display purposes at the voxel spacing reported in the acquisition parameters. Although a general consistency was observed between limiting spatial resolution measured by the $10 \%$ MTF and visual inspection of the bar patterns, it is clear that the reported voxel size (reconstructed voxel size) provides only a crude prediction of the spatial accuracy. ${ }^{9}$ It must be noted that the MTF measurement can be influenced by a number of factors, such as the difference in voxel values for the steel wire and the surrounding air, and the presence of small streak artifacts, ${ }^{9}$ as well as the selected ROI for PSF measurement.

The dose distributions were not uniform for all measurements, because the device does about a half rotation instead of a full rotation around the patient's head. In addition, the center of the FOV does not align with the center of the patient's head for FOVs with less than a $17-\mathrm{cm}$ diameter. As seen in Fig. 10, a symmetric dose gradient is evident for $17 \times 11 \mathrm{~cm}^{2}$ and $17 \times 6 \mathrm{~cm}^{2}$ FOVs. The $10 \times 10 \mathrm{~cm}^{2}, 10 \times 5 \mathrm{~cm}^{2}, 8 \times 8 \mathrm{~cm}^{2}$, and $5 \times 5 \mathrm{~cm}^{2}$ FOVs have off-axis positioning of the FOV to image specific portions of the mandible or maxilla, leading to more localized dose within the FOV. The DI1 values differed with different protocols, with an increase in the measured DI1 for larger FOVs for a given patient setting. The DI1 was expected to increase from a child to a large adult for a given FOV; however, for $10 \times 5$ and $10 \times 10 \mathrm{~cm}^{2}$ FOVs, DI1s were larger for the small adult than the average adult. This anomaly 


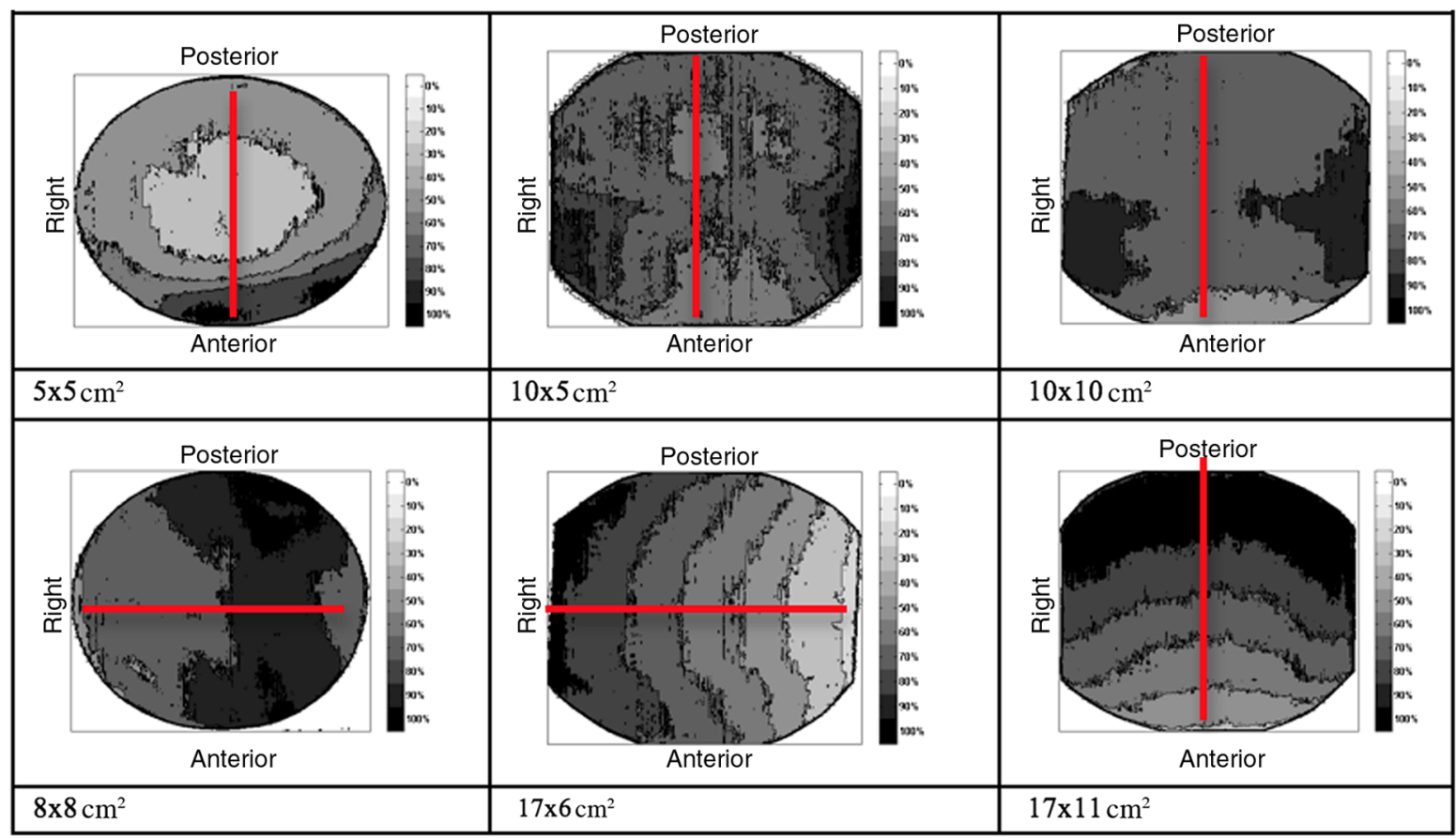

Fig. 10 Axial dose distribution for different FOVs. The dose distribution was obtained just for average adult and child settings. The red line represented the measuring diameter for DI1. The bottom and left side of the dose distribution correspond to the anterior and right side of the patient. The scale bar shows the distribution of dose as a percentage of maximum dose in each image.

can be due to positioning the ionization chamber slightly above or below the central plane in the $z$-axis for the different FOV acquisitions, which indicates that a range of acceptable values may be needed for dose measurements in a quality assurance program to account for small variations in ion chamber position within the phantom and within the FOV. The child settings did provide a reduction in the radiation dose to the patient, as suggested by Choi et al. ${ }^{23}$ and the Image Gently Campaign. ${ }^{24}$ Since the imaging technique changes $(\mathrm{kVp}, \mathrm{mA}$, exposure time) in addition to the FOV size between these two acquisition settings, the dose received by the patient can only be predicted by accounting for all factors and is not necessarily reduced by simply reducing the FOV size.

In this study, IQ metrics were compared for different patient imaging protocols. We found that the pediatric protocols did reduce the dose to the patient, with only small changes to

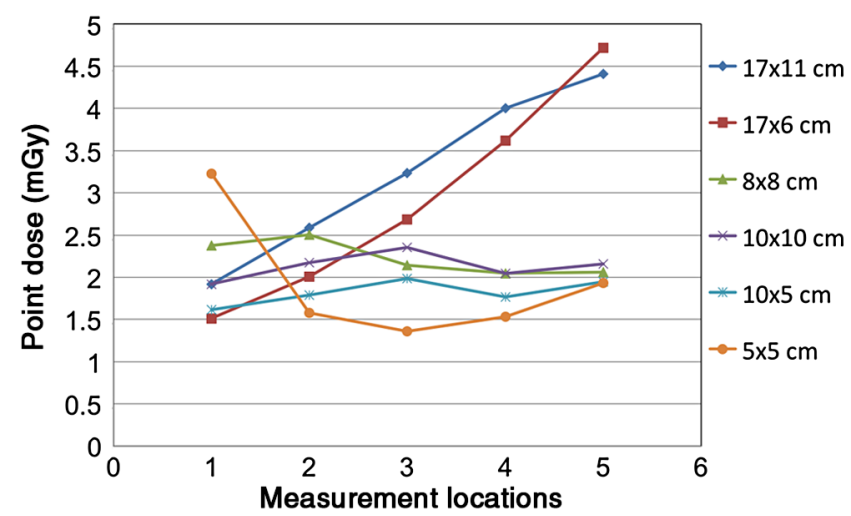

Fig. 11 The average of two dose readings at each point along the measuring diameter for average protocols is plotted. Points $1-5$ were placed anterior to posterior uniformly. the IQ - primarily an increase in image noise. This result suggests that using protocols that are specifically designed for smaller patients will reduce their radiation risk with no loss of image information. We also compared different imaging FOV, while maintaining similar reconstructed resolution settings (180 to $250 \mu \mathrm{m}$ ). Using a smaller FOV will reduce the amount of tissue exposed to radiation, thereby decreasing the dose. Furthermore, improvements in the image noise and the CNR were measured in the smaller FOV images, which may lead to improved visualization of important structures. Since the smaller FOV also has a higher resolution setting (not used in this study), which would increase the dose to the patient considerably if used, careful consideration of the imaging task must be taken to determine if the improved CNR of the small FOV is sufficient for the diagnostic task or if higher resolution imaging would be required.

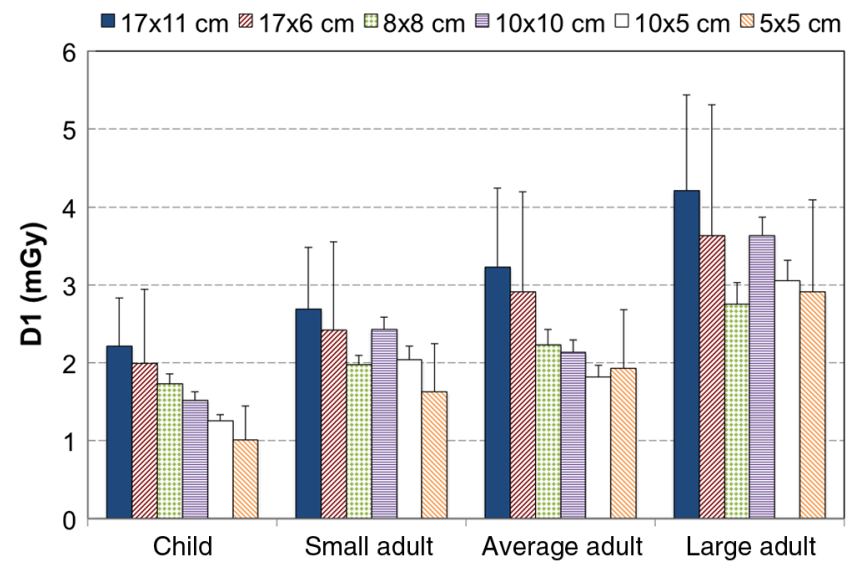

Fig. 12 The DI1 measured for different FOVs and different patient settings (child, small, average, and large adult). 


\section{Conclusions}

In this study, a complete characterization of the IQ and dose for a single dental CBCT unit was performed. All FOV and patient sizes were used to quantify the baseline values of a variety of IQ metrics that could be used in a quality assurance program. The measurement reproducibility and variability were investigated and found to be within $6 \%$ for the semiautomated methods used for all tests except artifact evaluation, which seems quite favorable for routine quality assurance. Images obtained with the child setting exhibited slightly reduced CNR and increased noise $(\sim 3 \%)$ compared to the adult settings, although there was a corresponding decrease in radiation dose, ranging from 0.8 to $1.0 \mathrm{mGy}$ compared to the average adult. In clinical practice, this degradation in IQ may not materialize, as the child and small adult settings would only be used with smaller patients.

\section{Acknowledgments}

The authors acknowledge Dr. Pierre Deman for assistance with the dosimetry measurements. Funding for this study was from an NSERC Discovery Grant (RGPIN 341581-10), CIHR Health Professional Student Research Award (2013), UBC Faculty of Dentistry S. Wah Leung Award (2013), and UBC Faculty of Dentistry Research Equipment Award (2012).

\section{References}

1. K. Horner, R. Jacobs, and R. Schulze, "Dental CBCT equipment and performance issues," Radiat. Prot. Dosim. 153(2), 212-218 (2013).

2. X. Liang et al., "A comparative evaluation of cone beam computed tomography (CBCT) and multi-slice CT (MSCT) Part I. On subjective image quality," Eur. J. Radiol. 75(2), 265-269 (2010).

3. M. Loubele et al., "Comparative study of image quality for MSCT and CBCT scanners for dentomaxillofacial radiology applications," Radiat. Prot. Dosim. 129(1-3), 222-226 (2008).

4. M. Loubele et al., "Image quality vs radiation dose of four cone beam computed tomography scanners," Dentomaxillofac. Radiol. 37(6), 309-318 (2008).

5. A. Suomalainen et al., "Dosimetry and image quality of four dental cone beam computed tomography scanners compared with multislice computed tomography scanners," Dentomaxillofac. Radiol. 38(6), 367-378 (2009).

6. J. P. Dillenseger et al., "MSCT versus CBCT: evaluation of high-resolution acquisition modes for dento-maxillary and skull-base imaging," Eur. Radiol. 25(2), 505-515 (2015).

7. L. Y. Du et al., "A quality assurance phantom for the performance evaluation of volumetric micro-CT systems," Phys. Med. Biol. 52(23), 7087-7108 (2007).

8. C. Steiding, D. Kolditz, and W. A. Kalender, "A quality assurance framework for the fully automated and objective evaluation of image quality in cone-beam computed tomography," Med. Phys. 41(3), 031901 (2014).

9. R. Pauwels et al., "Development and applicability of a quality control phantom for dental cone-beam CT," J. Appl. Clin. Med. Phys. 12(4), 3478 (2011).

10. J. W. Choi et al., "Relationship between physical factors and subjective image quality of cone-beam computed tomography images according to diagnostic task," Oral Surg. Oral Med. Oral Pathol. Oral Radiol. 119(3), 357-365 (2015).
11. G. R. Torgersen et al., "A phantom for simplified image quality control of dental cone beam computed tomography units," Oral Surg. Oral Med. Oral Pathol. Oral Radiol. 118(5), 603-611 (2014).

12. O. Gayou, "Influence of acquisition parameters on MV-CBCT image quality," J. Appl. Clin. Med. Phys. 13(1), 3638 (2012).

13. R. Pauwels et al., "Quantification of metal artifacts on cone beam computed tomography images," Clin. Oral Implants Res. 24(Suppl. A100), 94-99 (2013).

14. D. A. Jaffray and J. H. Siewerdsen, "Cone-beam computed tomography with a flat-panel imager: initial performance characterization," Med. Phys. 27(6), 1311-1323 (2000).

15. J. Bamba et al., "Image quality assessment of three cone beam CT machines using the SEDENTEXCT CT phantom," Dentomaxillofac. Radiol. 42(8), 20120445 (2013).

16. Sedentex Project, "Cone beam CT for dental and maxillofacial radiology," European Commission (2012).

17. J. A. Bryant, N. A. Drage, and S. Richmond, "Study of the scan uniformity from an i-CAT cone beam computed tomography dental imaging system," Dentomaxillofac. Radiol. 37(7), 365-374 (2008).

18. K. Kobayashi et al., "Accuracy in measurement of distance using limited cone-beam computerized tomography," Int. J. Oral Maxillofac. Implants 19(2), 228-231 (2004).

19. D. B. Plewes and P. B. Dean, "The influence of partial volume averaging on sphere detectability in computed tomography," Phys. Med. Biol. 26(5), 913-919 (1981).

20. W. De Vos, J. Casselman, and G. R. Swennen, "Cone-beam computerized tomography (CBCT) imaging of the oral and maxillofacial region: a systematic review of the literature," Int. J. Oral Maxillofac. Surg. 38(6), 609-625 (2009).

21. D. Brullmann and R. K. Schulze, "Spatial resolution in CBCT machines for dental/maxillofacial applications-what do we know today?" Dentomaxillofac. Radiol. 44(1), 20140204 (2015).

22. R. Pauwels et al., "Comparison of spatial and contrast resolution for cone-beam computed tomography scanners," Oral Surg. Oral Med. Oral Pathol. Oral Radiol. 114(1), 127-135 (2012).

23. E. Choi and N. L. Ford, "Measuring absorbed dose for i-CAT CBCT exams in child, adolescent and adult phantoms," Dentomaxillofac. Radiol. 44, 20150018 (2015).

24. M. J. Goske et al., "The 'image gently' campaign: increasing CT radiation dose awareness through a national education and awareness program," Pediatr. Radiol. 38(3), 265-269 (2008).

Elham Abouei received her BS degree in physics from the Sharif University of Technology, Iran, and her MS degree in physics in the field of medical physics from the University of British Columbia (UBC), Canada. She is a PhD candidate in the physics department at UBC. Her current research interests include medical image processing and optical cancer imaging.

Sierra Lee is an undergraduate student in the Doctor of Dental Medicine (DMD) program at the University of British Columbia. She received her BSc degree in mathematics from the University of British Columbia in 2010. Her current research interests include clinical applications of panoramic radiography and CBCT in dental practices.

Nancy L. Ford is an assistant professor in the Department of Oral Biological and Medical Sciences, and the director of the UBC Centre for High-Throughput Phenogenomics. She is an associate member of the Department of Physics and Astronomy at the University of British Columbia. She holds a $\mathrm{PhD}$ in medical biophysics from the University of Western Ontario. Her research interests include preclinical micro-CT and synchrotron imaging and clinical CBCT for medical and dental applications. 